\title{
Comparison of Cycle Outcomes among Women with Endometrioma or Non-Functional Cysts Versus Control
}

\author{
Enis OZKAYA ${ }^{1}$, Ebru COGENDEZ ${ }^{1}$, Elif TOZKIR ${ }^{1}$, Saadet UNSAL ${ }^{1}$, Ahmet YAVUZ ${ }^{1}$, Gulden TUNALI², \\ Ozgur UZUN³ , Evrim BOSTANCI ERGEN', Semra KAYATAS ESER ${ }^{1}$
}

Istanbul, Turkey

\begin{abstract}
OBJECTIVE: This study aimed to compare cycle outcomes among women with endometrioma or nonfunctional cysts versus control.

STUDY DESIGN: Women under 35 years of age with unexplained infertility underwent in vitro fertilization cycle. Two hundred and ninety-nine women were included in this study. Study groups consist of women with endometrioma $(n=82)$ or non-functional ovarian cysts $(n=100)$ and control group $(n=117)$. Women in each group were selected consecutively. Groups were matched for age and ovarian reserve and compared in terms of in vitro fertilization cycle outcome.

RESULTS: There were no significant differences among groups in terms of mean age, early follicular phase Follicle-Stimulating Hormone, and anti-müllerian hormone levels $(p>0.05)$. Baseline total antral follicle counts were similar among groups $(p>0.05)$. There were significant differences among groups in terms of mean starting and total gonadotropin dose, peak estradiol level, total oocyte number, and mature oocyte numbers $(p<0.05)$. Clinical pregnancy rates were $10.7 \%, 17.3 \%$, and $31.6 \%$ in groups with non-functional cyst, endometrioma and control group respectively $(p<0.05)$. The presence of a non-functional cystic mass of ovary on the starting day of stimulation was a risk factor for poor cycle outcome in terms of clinical pregnancy rates [OR=0.5 (95\% Cl; 0.2-0.9, p=0.03)].
\end{abstract}

CONCLUSION: Our data showed that the presence of any kind of cystic mass of the ovary especially the non-functional cysts on the starting day of stimulation was a risk factor for poor cycle outcomes in terms of clinical pregnancy rates.

Keywords: Cysts, Endometrioma, In vitro fertilization, The clinical pregnancy rate

Gynecol Obstet Reprod Med 2021;27(3):254-259

${ }^{1}$ Department of Obstetrics and Gynecology, Health Sciences University Zeynep Kamil Women and Children's Diseases Training and Research Hospital İstanbul, Turkey

2 Department of Histology and Embryology, Health Sciences University Zeynep Kamil Women and Children's Diseases Training and Research Hospital Istanbul, Turkey

${ }^{3}$ Department of Histology and Embryology, Istanbul University-Cerrahpasa Medical School Ístanbul, Turkey

Address of Correspondence: Enis Ozkaya

Department of Obstetrics and Gynecology,

Health Sciences University Zeynep Kamil

Women and Children's Diseases Training and Research Hospital, Uskudar, Istanbul enozkaya1979@gmail.com

SSubmitted for Publication 30.05.2020 Revised for Publication: 21.07.2020 Accepted for Publication: 26.10.2020 Online Published: 15.01.2021

ORCID IDs of the authors: EC: 0000-0001-7062-3076, $S U: 0000-0001-7953-9866$, GT: 0000-0001-8816-1295,

EO: 0000-0001-6580-1237,

ET: 0000-0001-6996-4615,

AY: 0000-0001-8171-2146,

OU: 0000-0002-2194-1825,

EBE: 0000-0002-1634-6781, SKE: 0000-0003-4675-808X

\begin{tabular}{|c|c|}
\hline \multirow{3}{*}{$\begin{array}{c}\text { Quick Response Code: } \\
\text { 品 } \\
\end{array}$} & Access this article online \\
\hline & $\begin{array}{l}\text { Website: www.gorm.com.tr } \\
\text { e- mail: info@gorm.com.tr }\end{array}$ \\
\hline & DOI:10.21613/GORM.2020.1110 \\
\hline
\end{tabular}

How to cite this article: Ozkaya E. Cogendez E. Tozkir E. Unsal S. Yavuz A. Tunali G.Uzun O. Bostanci Ergen E. Kayatas Eser S. Comparison of Cycle Outcomes Among Women with Endometrioma or Non-Functional Cysts Versus Control. Gynecol Obstet Reprod Med. 2021;27(3):254-259

\section{Introduction}

Ovarian cysts are among the most common disorders in gynecology and infertility outpatient clinics in women of reproductive age. The most common cysts in infertile women, independent of the ovarian reserve, are simple anechoic cysts and endometriomas (1). Whether cysts negatively affect in vitro fertilization (IVF) prognosis and how aspiration or surgical treatment of these cysts affect IVF results are still challenging today. Today, the preferred methods of managing ovarian cysts at the onset of IVF cycles include the expectant approach, combined oral contraceptive use, continuation of gonadotropin-releasing hormone analog (GnRH-a) suppression, cyst aspiration, and direct initiation of gonadotropins by ignoring cysts $(2,3)$. The effects of ovarian cysts at the onset of the cycle on IVF results are explained by three mechanisms. These mechanisms are a significant increase in estradiol (E2) levels caused by the cyst, respectively, by suppressing the FollicleStimulating Hormone (FSH) levels, leading to a decrease in follicular recruitment, narrowing the ovarian surface area due to the mass effect of the cysts, preventing the growth of follicles and reducing the number and quality of oocytes collected 
by disrupting the blood flow to the follicles. $(4,5)$. When we look at the literature, besides the publications reporting that simple anechoic cysts detected at the beginning of the cycle did not negatively affect the IVF results $(6,7)$; there are also publications claiming to affect IVF results negatively $(8,9)$. Weak follicular recruitment and increased cycle cancellation rates have been proposed concerning these negative results $(8,9)$. The situation is a bit more complicated for endometriomas. Whether IVF failure is caused by endometriosis or endometriosis disease itself remains current among controversial issues in the literature. Also, considering that surgical excision of endometriomas further reduces ovarian reserve, more attention should be paid to the approach to endometriomas detected initially in women who are directed go IVF (10).

This study aims to investigate the effect of non-functional ovarian cyst and endometriomas, detected at the beginning of fresh IVF - ET cycles, on cycle outcome.

\section{Material and Method}

A total of 299 consecutive women with a normal ovarian reserve and $<35$ years of age who had undergone IVF/ICSI treatment in Health Sciences University Zeynep Kamil Women and Children's Health Training and Research Hospital and had an ovarian endometrioma diagnosed by ultrasound $(n=82)$ or had a basal simple ovarian cyst at the beginning of ovarian stimulation $(n=100)$ or a group of women without any baseline cyst (control group, $n=117$ ) were retrospectively identified. This retrospective, case-control study was carried out between May 2018 and December 2019. Our institutional ethics committee acknowledges that the study does not require a formal ethical review because of the retrospective review of medical records accepted by all patients and the study was conducted in accordance with the Declaration of Helsinki.

The study groups consisted of 82 women with unilateral ovarian endometriomas of $10-50 \mathrm{~mm}$, diagnosed by transvaginal ultrasound or baseline simple ovarian cysts $(n=100)$. The control group included 117 women who had no baseline ovarian cysts. Simple ovarian cysts $(10-35 \mathrm{~mm})$ were defined as echo-free cysts with regular borders with normal tumor markers (11). The male-factor was the only main cause of infertility in the control group. A transvaginal ultrasound scan was performed and a blood sample was taken on the second or third day of the menstrual bleed. Average serum estradiol concentrations in all three groups were $<50 \mathrm{pg} / \mathrm{ml}$ on the first day of stimulation and progesterone level $<1.5 \mathrm{ng} / \mathrm{mL}$. Patients with high FSH levels (>10 IU/1), age 40 and older were excluded.

For all participants, a gonadotropin-releasing hormone antagonist protocol was used for IVF/ICSI. A regimen of daily recombinant follicle-stimulating hormone ( $\mathrm{rFSH}$; Gonal-F, Merck-Serono, Geneva, Switzerland) was started on the second day of the menstrual cycle. The dose used ranged from
150 IU to 300 IU and was determined by each patient's basal clinical characteristics. Mean follicular growth was monitored every 2-3 days via two-dimensional transvaginal sonography. The daily dose of rFSH was adjusted from day 5 of stimulation according to the ovarian response. The antagonist (Cetrorelix, Merck-Sereno, Geneva, Switzerland) was administered at a dose of $0.25 \mathrm{mg} /$ day when the follicular size reached $12 \mathrm{~mm}$. When the follicular size reached $18 \mathrm{~mm}, 250$ $\mu \mathrm{g}$ recombinant human chorionic gonadotropin (hCG; Ovitrelle, Merck-Sereno, Geneva, Switzerland) was administered subcutaneously, and follicular puncture was performed after 34-36 hours. Next, 8\% vaginal progesterone gel (Crinone gel $8 \%$; Merck-Sereno, Geneva, Switzerland) was applied twice daily. ICSI was applied for each oocyte obtained by follicular puncture. Elective transfer of single grade1 or $2 \mathrm{em}$ bryos was performed either at cleavage (day 3) or blastocyst (day 5) stage, according to the developmental characteristics of the embryo. Prior to the transfer, all embryos were graded and given a rating according to blastomere quality as follows: Grade I, equal-sized symmetrical blastomeres with $<10 \%$ fragmentation were given a rating of 4 ; Grade II, uneven blastomeres with $<10 \%$ fragmentation were rated as 3; Grade III, $10 \%$ to $50 \%$ fragmentation were rated as 2 ; and Grade IV, $>50 \%$ fragmentation were given a rating of 1 . All embryos were assessed by one embryologist. Serum levels of the $\beta$-subunit of hCG $(\beta$-hCG) were measured after 2 weeks. Ultrasonography was performed to detect the pulse of the fetus and confirm a clinical pregnancy.

These three groups were compared for differences in the number of oocytes retrieved, oocyte quality as a ratio of mature oocytes, embryo quality, and clinical pregnancy rate.

Statistical analyses were carried out with SPSS Version 12.0 (SPSS Inc., Chicago, Ill., USA). Statistically significant differences were determined using the Kruskal Wallis test. Categorical variables were compared by the Chi-square test. Statistical significance was defined as $p<0.05$.

\section{Results}

There were no significant differences among groups in terms of mean age, early follicular phase FSH and anti-müllerian hormone $(\mathrm{AMH})$ levels (Table I, $p>0.05)$. Baseline total antral follicle counts were similar among groups $(p>0.05)$. There were significant differences among groups in terms of mean starting and total gonadotropin dose, peak estradiol level, total oocyte number, and mature oocyte numbers (Table II, $p<0.05)$. Although the rates of transferred embryo grades were not statistically different among groups $(81.2 \%$ Grade I, $18.8 \%$ Grade II in control, $73.2 \%$ Grade I, 26.8\% Grade II in endometrioma group, $82 \%$ Grade I, 18\% Grade II in the group with non-functional cysts, $p>0.05$ ), clinical pregnancy rates were $10.7 \%, 17.3 \%$ and $31.6 \%$ in groups with non-functional cyst, endometrioma, and control group respectively $(p<0.05)$. 
Table I: Comparison summary of baseline characteristics of groups

\begin{tabular}{lrrrrrcc}
\hline & \multicolumn{2}{c}{ Control } & \multicolumn{2}{c}{ Endometrioma } & \multicolumn{2}{c}{ Simple ovarian cysts } & $p$ \\
\cline { 2 - 6 } & Mean & SD & Mean & SD & Mean & SD \\
\hline Age (years & 31.1 & 3.3 & 31.3 & 2.9 & 31.3 & 3.6 & 0.8 \\
FSH (IU/l) & 6.1 & 2.1 & 6.4 & 2.4 & 6.9 & 3.1 & 0.1 \\
Estradiol (pg/mL) & 40.1 & 14.5 & 45.7 & 15.9 & 45.6 & 16.8 & 0.03 \\
AMH (ng/mL) & 2.4 & 1.8 & 2.2 & 1.2 & 2.3 & 1.3 & 0.7 \\
AFC & 11.9 & 3.9 & 11.1 & 3.6 & 11.9 & 4 \\
\hline
\end{tabular}

SD: Standard deviation, FSH: Follicle-stimulating hormone, AMH: Anti-müllerian hormone, AFC: Antral follicle count

Table II: Comparison summary of cycle characteristics of groups

\begin{tabular}{lcccrrrr}
\hline & \multicolumn{2}{c}{ Control } & \multicolumn{2}{c}{ Endometrioma } & \multicolumn{2}{c}{ Simple ovarian cysts } \\
\hline & Mean & SD & Mean & SD & Mean & SD \\
\hline Starting gonadotropin dose $(\mathrm{u})$ & 231.410 & 67.8 & 272.866 & 58.8 & 273.750 & 52.6 & $<0.001$ \\
Total gonadotropin dose $(\mathrm{u})$ & 2129.1 & 776.7 & 2839.9 & 1072.8 & 2551.4 & 800.9 & $<0.001$ \\
Peak estradiol level $(\mathrm{pg} / \mathrm{mL})$ & 1702.9 & 989.6 & 1765.4 & 1026.4 & 1416.9 & 960.9 & 0.03 \\
\# of total oocytes retrieved & 8.14 & 5.230 & 7.29 & 4.867 & 6.19 & 5.114 & 0.005 \\
\# of mature oocytes retrieved & 6.52 & 4.238 & 5.06 & 3.546 & 4.40 & 3.164 & 0.001 \\
\hline
\end{tabular}

SD: Standard deviation

Two-group comparisons revealed significantly different clinical pregnancy rates between groups with endometrioma and control $(p=0.03)$, groups with a non-functional cyst, and control $(p=0.01)$. On the other hand, no significant differences were determined between the endometrioma group and group with a non-functional cyst $(p>0.05)$. The presence of a nonfunctional cystic mass of ovary on the starting day of stimulation was a risk factor for poor cycle outcome in terms of clinical pregnancy rates $[\mathrm{OR}=0.5(95 \% \mathrm{CI} ; 0.2-0.9, p=0.03)]$. The mean cyst diameter was significantly higher in the group with functional cysts $(20.5 \mathrm{~mm}$ vs. $31.6 \mathrm{~mm}, p<0.001)$. Multivariate regression analysis showed that groups were not significantly associated with the clinical pregnancy when adjusted for cyst diameter (constant $=0.012, p>0.05$ ).

\section{Discussion}

In this study, we aimed to compare cycle outcomes among women with endometrioma or non-functional cysts versus control. Our data showed that on the day of stimulation, any cystic ovarian mass, especially the presence of dysfunctional cysts, is a risk factor for poor cycle outcome in terms of clinical pregnancy rates. The presence of a non-functional cystic mass of ovary on the starting day of stimulation was a risk factor for poor cycle outcome in terms of clinical pregnancy rates $[\mathrm{OR}=0.5(95 \% \mathrm{CI} ; 0.2-0.9, p=0.03)]$.

In recent years, there is widespread consensus on not removing endometriomas before the IVF, as clearly stated in both the American Association for Reproductive Medicine (ASRM) and the European Society for Human Reproduction and Embryology (ESHRE) $(10,12,13)$.
However, a conservative approach to endometriomas can pose some risks such as decreased ovarian response potential, difficulty in oocyte pick up procedure, and the risk of missing possible adnexal malignancy presumed to be benign cysts $(10,14)$. There is no consensus yet on the mechanism of endometriomas causing infertility. However, the researchers reported that the presence of endometrioma causes an increase in oxidative stress in the follicular environment and changes the iron metabolism, and affects ovarian physiology $(15,16)$. There are publications that endometriomas reduce the number of oocytes collected $(17,18)$, oocyte quality, embryo quality, and implantation rate $(18,19)$. In a study of Yang et al., the presence of endometrioma at the onset of the cycle showed a negative effect on the number of oocytes collected, the number of MII oocytes and the number of embryos while implantation rate, clinical pregnancy and live birth rates are similar to those without endometriomas (20). In the study conducted by Kumbak et al. in 2007 with normoresponder cases, the effect of the presence of endometrioma on IVF results during the IVF cycle was evaluated and this was not shown to affect pregnancy success, but with lower embryo quality and lower implantation rates. The authors argued that this negative effect in the study occurred not as a result of the mass presence, but as a result of the disease itself (21). In the study of Ashrafi et al. included in the meta-analysis of Somigliana in 2015, fewer oocytes were collected in cases with $<3 \mathrm{~cm}$ endometrioma and the number of mature oocytes was lower than in those without endometriomas; however, it has been reported that the total number of embryos transferred per patient, embryo quality, and pregnancy rate are not affected $(22,23)$. However, in our study, we found that endometriomas detected at the beginning of the cycle lead to poor ovarian response (number of oocytes collected), but also significantly decrease the rate of clinical 
pregnancy. In our study, this effect was more clearly observed in non-functional ovarian cysts. Many studies have been conducted on the effects of cysts detected at the beginning of the IVF cycle on gonadotropin doses used during the cycle, the number of oocytes collected, and pregnancy rates. An initially detected ovarian cyst may be endometrioma or malignant, as well as the persistence of the follicle or corpus luteum cyst formed in the previous cycle. It is known that functional cysts occur due to the increase of gonadotropin secondary to the flare-up effect of the analog in IVF cycles where GnRH analogs are used (4). These functional cysts detected at the beginning of the cycle may cause early luteinizing hormone surge and progesterone elevation, leading to poor oocyte quality and poor endometrial development. In the systematic review of McDonnell et al., GnRH-a therapy was compared with cyst aspiration performed for functional ovarian cysts $>2$ $\mathrm{cm}$ at the beginning of the IVF cycle. As a result of this review, the authors stated that cyst aspiration is not superior to GnRH-a therapy in terms of live birth, clinical pregnancy, number of recruited follicles, and number of oocytes collected; in addition, they reported that cyst aspiration has disadvantages such as the need for anesthesia and the risk of surgical complications (3). Qublan et al. investigated the effects of functional ovarian cysts on IVF treatment outcomes; in their study, they showed that the presence of cyst was associated with higher cycle cancellation rates and lower implantation and pregnancy rates compared to cases without ovarian cysts (24). In the same study, it was reported that cyst aspiration did not improve the IVF result. Besides studying only functional cysts, another difference of this study from our study is that bilateral ovarian cysts are also included in the study of Qublan et al. Pereira et al. compared cyst aspiration and GnRH antagonist administration, and both methods were reported to be acceptable in terms of their effects on IVF cycle results in ovarian cyst management detected at the beginning of IVF treatment (25). Segal et al. evaluated the effect of baseline simple ovarian cysts on IVF results, and showed that these cysts significantly reduced the rate of pregnancy in IVF cycles. In this study of Segal, the authors suggested that these cysts prevent the development of other follicles by mechanical action or negatively affect folliculogenesis by disrupting local follicular angiogenesis (1). In the study of Segal, possible endometriomas were also included in the analysis since simple and complex cysts at the beginning of the cycle were not clearly distinguishable from each other. This further strengthens the accuracy of our results. Because we think that both simple cysts and endometriomas both impair angiogenesis by mass effect and this negatively affects IVF results. In the 2009 study of Kumbak et al., direct stimulation to simple ovarian cysts detected at the beginning of the cycle in normoresponder cases was compared with the stimulation following serum estradiol level decrease after cyst aspiration. The authors argued at the end of the study that the ovarian cysts at the beginning of the cycle did not interfere with IVF stimulation, and the stimulation initiated after the serum estradiol levels were decreased by cyst aspiration did not show a favorable result (26). In our study, we determined that both non-functional anechoic cysts and endometriomas detected at the beginning of the cycle are poor prognostic factors for the ovarian response. The difference in our study from Kumbak and colleagues is that the cysts detected at the beginning of the cycle are non-functional and the endometrioma group is also included in the study. Functional ovarian cysts have been studied in most studies. In our clinic, we apply combined oral contraceptives to $>2 \mathrm{~cm}$ functional cysts, while expecting an $<2 \mathrm{~cm}$ functional ovarian cyst. As a result of these treatments, we start the IVF cycle when the cyst resolves and the estradiol level is $<50 \mathrm{pg} / \mathrm{mL}$ and progesterone level $<1.5 \mathrm{ng} / \mathrm{mL}$. In our study, we disabled one of the mechanisms explaining the possible harmful effects of ovarian cysts at the onset of the cycle on the results of IVF-ET cycles by studying non-functional cysts. Early LH surge due to estradiol increase observed in functional cysts and high endometrial progesterone exposure are the possible underlying mechanisms for the poor outcome of the functional cysts, by studying non-functional cysts we eliminated this negative effect. As a result of our study, nonfunctional ovarian cysts, which are thought to be more innocent, may also affect the IVF cycle results negatively and reduces the rate of clinical pregnancy. This effect was also valid for endometriomas in our study. Our clinical pregnancy rate is $10.7 \%$ in the non-functional cyst group, $17.3 \%$ in endometrioma cases, $29.9 \%$ in the group without cysts. As a result, these results led us to consider that non-functional ovarian cysts and endometriomas occupy the area covered by mechanical action which can disrupt folliculogenesis and adversely affect IVF outcome parameters (decreased oocyte count and oocyte quality, low clinical pregnancy rate), additionally, the majority of these simple cysts are persistent corpus luteum, although we measured progesterone levels at the early follicular phase, we do not measure other paracrine, autocrine hormones which may interfere with the endometrial receptivity such as PGF2alfa which has been shown to be decreased in such cases and has a significant role in endometrial receptivity (27). For these reasons, the cancellation of the cycle in cases with a simple cyst of $<5 \mathrm{~cm}$ encountered at the beginning of the cycle regardless of the ovarian reserve may be suggested based on these results; additionally, surgical excision may be beneficial in patients with normal ovarian reserve with endometrioma $<4$ $\mathrm{cm}$ before the cycle. However, there is still a need for more well-designed, multicenter, prospective randomized controlled trials.

Major drawbacks in this study were the retrospective nature of this study and the relatively small sample size.

In conclusion, the presence of any kind of cystic mass of the ovary especially the non-functional cysts on the starting day of stimulation was a risk factor for poor cycle outcomes in terms of clinical pregnancy rates. 
Acknowledgment: None

Conflict of Interest: Authors have nothing to declare

Funding: None

Author contributions: EO: Concept, data analysis, manuscript preparation, EC: Manuscript preparation, ET: Data collection, SU: Data collection, AY: Data collection, GT: Data collection, OU: Data collection, EBE: Data collection, SKE: Supervision.

\section{References}

1. Wahd SA, Alalaf SK, Al-Shawaf T, Al-Tawil NG. Ovarian reserve markers and assisted reproductive technique (ART) outcomes in women with advanced endometriosis. Reprod Biol Endocrinol. 2014;12:120. Doi: 10.1186/1477-7827-12-120.

2. Segal S, Shifren JL, Isaacson KB, Leykin L, Chang Y, Pal L, et al. Effect of a baseline ovarian cyst on the outcome of in vitro fertilization-embryo transfer. Fertil Steril. 1999;71(2):274-7. Doi: 10.1016/s0015-0282(98)00449-x.

3. Biljan MM, Lapensée L, Mahutte NG, Bissonnette F, Hemmings R, Tan SL. Effects of functional ovarian cysts detected on the 7 th day of gonadotropin-releasing hormone analog administration on the outcome of IVF treatment. Fertil Steril. 2000;74(5):941-5. Doi: 10.1016/ s0015-0282(00)01555-7.

4. McDonnell R, Marjoribanks J, Hart RJ. Ovarian cyst aspiration prior to in vitro fertilization treatment for subfertility. Cochrane Database Syst Rev. 2014;2014(12): CD005999. Doi: 10.1002/14651858.CD005999.pub2.

5. Firouzabadi RD, Sekhavat L, Javedani M. The effect of ovarian cyst aspiration on IVF treatment with GnRH. Arch Gynecol Obstet. 2010;281(3):545-9. Doi: 10.1007/ s00404-009-1195-9.

6. Hornstein MD, Barbieri RL, Ravnikar VA, McShane PM. The effects of baseline ovarian cysts on the clinical response to controlled ovarian hyperstimulation in an in vitro fertilization program. Fertil Steril. 1989;52(3):43740. Doi: 10.1016/s0015-0282(16)60914-7.

7. Stewart EA, Jackson KV, Friedman AJ, Rein MS, Fox JH, Hornstein MD. The effect of baseline complex ovarian cysts on in vitro fertilization outcome. Fertil Steril. 1992; 57(6):1274-8. Doi: 10.1016/S0015-0282(16)55087-0.

8. Thatcher SS, Jones E, DeCherney AH. Ovarian cysts decrease the success of controlled ovarian stimulation and in vitro fertilization. Fertil Steril. 1989;52(5):812-6. Doi: 10.1016/s0015-0282(16)61036-1.

9. Goldberg JM, Miller FA, Friedman CI, Dodds WG, Kim $\mathrm{MH}$. Effect of baseline ovarian cysts on in vitro fertilization and gamete intrafallopian transfer cycles. Fertil Steril. 1991;55(2):319-23. Doi: 10.1016/s0015-0282(16)54123-5.

10. Garcia-Velasco JA, Somigliana E. Management of endometriomas in women requiring IVF: to touch or not to touch. Hum Reprod. 2009;24(3):496-501. Doi: 10.1093/ humrep/den398.

11. Wasnik AP, Menias CO, Platt JF, Lalchandani UR, Bedi DG, Elsayes KM. Multimodality imaging of ovarian cystic lesions: Review with an imaging based algorithmic approach. World J Radiol. 2013;5(3):113-25. Doi: 10.4329/ wjr.v5.i3.113

12. Dunselman GA, Vermeulen N, Becker C, Calhaz-Jorge C, D'Hooghe T, De Bie B et al. ESHRE guideline: management of women with endometriosis. Hum Reprod. 2014; 29(3):400-12. Doi: 10.1093/humrep/det457.

13. Garcia-Velasco JA, Mahutte NG, Corona J, Zúñiga V, Gilés J, Arici A, et al. Removal of endometriomas before in vitro fertilization does not improve fertility outcomes: a matched, case-control study. Fertil Steril. 2004;81(5): 1194-7. Doi: 10.1016/j.fertnstert.2003.04.006.

14. Somigliana E, Vigano' P, Parazzini F, Stoppelli S, Giambattista E, Vercellini P. Association between endometriosis and cancer: a comprehensive review and a critical analysis of clinical and epidemiological evidence. Gynecol Oncol. 2006;101(2):331-41. Doi: 10.1016/j. ygyno.2005.11.033.

15. Giacomini E, Sanchez AM, Sarais V, Beitawi SA, Candiani M, Viganò P. Characteristics of follicular fluid in ovaries with endometriomas. Eur J Obstet Gynecol Reprod Biol. 2017;209:34-8. Doi: 10.1016/j.ejogrb. 2016. 01.032 .

16. Nickkho-Amiry M, Savant R, Majumder K, Edi-O'sagie E, Akhtar M. The effect of surgical management of endometrioma on the IVF/ICSI outcomes when compared with no treatment? A systematic review and meta-analysis. Arch Gynecol Obstet. 2018;297(4):1043-1057. Doi: 10.1007/s00404-017-4640-1.

17. Suzuki T, Izumi S, Matsubayashi H, Awaji H, Yoshikata $\mathrm{K}$, Makino T. Impact of ovarian endometrioma on oocytes and pregnancy outcome in in vitro fertilization. Fertil Steril. 2005;83(4):908-13. Doi: 10.1016/j.fertnstert. 2004.

18. Yanushpolsky EH, Best CL, Jackson KV, Clarke RN, Barbieri RL, Hornstein MD. Effects of endometriomas on ooccyte quality, embryo quality, and pregnancy rates in in vitro fertilization cycles: a prospective, case-controlled study. J Assist Reprod Genet. 1998;15(4):193-7. Doi: 10. 1023/a:1023048318719.

19. Azem F, Lessing JB, Geva E, Shahar A, Lerner-Geva L, Yovel I, et al. Patients with stages III and IV endometriosis have a poorer outcome of in vitro fertilization-embryo transfer than patients with tubal infertility. Fertil Steril. 1999;72(6):1107-9. Doi:10.1016/s0015-0282(99)00392-1.

20. Yang C, Geng Y, Li Y, Chen C, Gao Y. Impact of ovarian endometrioma on ovarian responsiveness and IVF: a systematic review and meta-analysis. Reprod Biomed Online. 2015;31(1):9-19. Doi: 10.1016/j.rbmo.2015.03.005.

21. Kumbak B, Kahraman S, Karlikaya G, Lacin S, Guney A. In vitro fertilization in normoresponder patients with endometriomas: comparison with basal simple ovarian cysts. 
Gynecol Obstet Invest. 2008;65(3):212-6. Doi: 10.1159/ 000112310 .

22. Ashrafi M, Fakheri T, Kiani K, Sadeghi M, Akhoond MR. Impact of the endometrioma on ovarian response and pregnancy rate in in vitro fertilization cycles. Int J Fertil Steril. 2014;8(1):29-34. PMID: 24696766.

23. Somigliana E, Benaglia L, Paffoni A, Busnelli A, Vigano $\mathrm{P}$, Vercellini P. Risks of conservative management in women with ovarian endometriomas undergoing IVF. Hum Reprod Update. 2015;21(4):486-99. Doi: 10.1093/ humupd/dmv012.

24. Qublan HS, Amarin Z, Tahat YA, Smadi AZ, Kilani M. Ovarian cyst formation following GnRH agonist administration in IVF cycles: incidence and impact. Hum Reprod.
2006;21(3):640-4. Doi: 10.1093/humrep/dei371.

25. Pereira N, Amrane S, Hobeika E, Lekovich JP, Chung PH, Rosenwaks Z. Cyst aspiration or GnRH antagonist administration for ovarian cysts detected at the start of fresh in vitro fertilization cycles. Gynecol Endocrinol. 2016; 32(7):562-5. Doi: 10.3109/09513590.2016.1139565.

26. Kumbak B, Kahraman S. Management of prestimulation ovarian cysts during assisted reproductive treatments: impact of aspiration on the outcome. Arch Gynecol Obstet. 2009;279(6):875-80. Doi: 10.1007/s00404-008-0837-7.

27. Aksel S, Schomberg DW, Hammond CB. Prostaglandin F2alpha levels in human ovarian plasma in pregnancy and in a case of Halban's disease. Obstet Gynecol. 1978; 52(4):421-3. PMID: 714322. 